\title{
Müllerian Adenosarcoma Arising From Rectal Endometriosis
}

\author{
Chunseok Yang, Hoon Kyu $\mathrm{Oh}^{1}$, Daedong Kim \\ Department of Surgery, Catholic University of School of Medicine, Daegu; ${ }^{1}$ Department of Surgery, Daegu Catholic University Medical \\ Center, Daegu, Korea
}

\begin{abstract}
A Müllerian adenosarcoma is an extremely rare tumor characterized by a stromal component of usually low-grade malignancy and by a benign glandular epithelial component. A Müllerian adenosarcoma occurs mainly in the uterus, but also in extrauterine locations. Extrauterine Müllerian adenosarcomas are thought to arise from endometriotic deposits. A 36-year-old female presented to Daegu Catholic University Medical Center with a symptom of loose stool for several months. The imaging studies revealed a rectal mass, so she underwent a laparoscopic low anterior resection. Although extemporary pathology revealed an inflammatory myofibroblastic tumor, the final histologic diagnosis was a Müllerian adenosarcoma arising from rectal endometriosis. To our knowledge, except a concomitant rectal villotubular adenoma, cases of Müllerian adenosarcomas arising the rectal wall are rare. An adenosarcoma arising from endometriosis should be considered in the differential diagnosis of a pelvic mass, even one appearing in rectal wall, because ectopic endometrial tissue exists everywhere.
\end{abstract}

Keywords: Mullerian adenosarcoma; Endometriosis; Rectal neoplasms; Adenosarcoma

\section{INTRODUCTION}

A Müllerian adenosarcoma was first reported in 1974 by Clement and Scully to describe an unusual variant of a Müllerian mixed tumor of the uterus $[1,2]$. It is characterized by a mixture of a benign glandular epithelium and a malignant sarcomatous stroma, which are regarded as intermediate features between a benign adenofibroma and a carcinosarcoma. It occurs mainly in the uterus of postmenopausal women and also in young adults and in extrauterine locations.

An extrauterine Müllerian adenosarcoma is rare and is thought to arise in ectopic foci of endometriosis. The most common site of an extrauterine Müllerian adenosarcoma is the ovary, but other reported locations are the vagina, fallopian tube, peritoneal sites

Received: May 25, 2014 - Accepted: July 23, 2014

Correspondence to: Daedong Kim, M.D.

Department of Surgery, Daegu Catholic University Medical Center, Catholic University of Daegu School of Medicine, 33 Duryugongwon-ro 17-gil,

Nam-gu, Daegu 705-718, Korea

Tel: +82-53-650-4061, Fax: +82-53-624-7185

E-mail:ddkim@cu.ac.kr

(C) 2014 The Korean Society of Coloproctology

This is an open-access article distributed under the terms of the Creative Commons Attribution NonCommercial License (http://creativecommons.org/licenses/by-nc/3.0) which permits unrestricted noncommercial use, distribution, and reproduction in any medium, provided the original work is properly cited. such as the pouch of Douglas, intestinal serosa, and liver [3, 4]. We present a case of primary extrauterine Müllerian adenosarcoma arising from endometriosis of the rectum.

\section{CASE REPORT}

A 36-year-old female visited Daegu Catholic University Medical Center complaining of having had a loose stool for several months. She complained of dysmenorrhea, deep dyspareunia, and mild intermittent hematochezia. A Cesarean section had been performed 10 years earlier due to a narrow pelvis. Except these, she had no significant medical history or family history.

Rectal examination revealed a 3-cm-sized, fixed, round, lobulated, firm mass at the anterior side of the rectum, about $8 \mathrm{~cm}$ from the anal verge. Laboratory studies were unremarkable. Colonoscopy (Fig. 1) showed that a polypoid mass accounted for one-third of the diameter of the rectum, and biopsy revealed it to be a hyperplastic polyp. Computed tomography (Fig. 2A) showed a 2.7-cmsized mass at the anterior side of the rectum, and magnetic resonance imaging (Fig. 2B) showed intermediate high signal intensity on the T2-weighted image. Based on the patient's wish and clinical pictures, we advised a local excision.

Because of the high-lying tumor and its fixation to surrounding tissue, the patient underwent a transanal incisional biopsy. Frozen biopsy revealed inflammation with an ulcer, and permanent bi- 
Volume 30, Number 5, 2014

opsy revealed an inflammatory myofibroblastic tumor. A subsequent laparoscopic low anterior resection was performed to excise the tumor completely and to rule out a possible malignancy. Intraoperative findings showed that left fallopian tube was twisted and had adhesed to the lateral pelvic wall and that the uterus was thick, edematous and severely attached to the rectum.

The final slides showed a biphasic appearance of epithelial and stromal components. The excised rectum was a $11.5-\mathrm{cm}$-sized segment, and a 1:1 mounted H\&E stained section (Fig. 3) shows a $2.5 \times 2$-cm intramural nodular mass (arrow) with a polypoid mucosal protrusion (arrowhead). Multiple intramural and serosal endometriotic foci (circles) are also present. The histologic findings suggested a diagnosis of an adenosarcoma (Fig. 4). To identify the characteristics of the adenosarcoma, we performed immunohistochemistry on the endometriosis and the mural mass (Fig. 5).

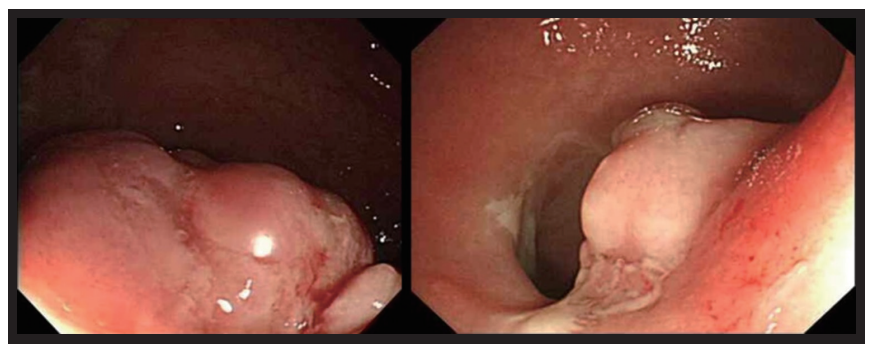

Fig. 1. A polypoid mass accounting for one-third of the diameter of the rectum was seen during the colonoscopy.
The postoperative clinical course of the patient was uneventful, and the patient did not undergo any cycle of chemotherapy or radiotherapy. We followed this patient for 5 years without any evidence of recurrence.

\section{DISCUSSION}

A Müllerian adenosarcoma is an uncommon mixed epithelialmesenchymal tumor of low-grade malignancy that affects the female genital tract [2]. Usually, postmenopausal women have a higher risk for a uterine Müllerian adenosarcoma compared with younger women, and its median age at diagnosis is 58 years [5]. However, extrauterine Müllerian adenosarcomas occur in younger women (mean age, 44 years) and are more aggressive than uterine tumors. Probably because the extrauterine location allows free access to the peritoneal cavity, the spread of an extrauterine Müllerian adenosarcoma may not be retarded by the thick myometrial wall. Therefore, for a uterine Müllerian adenosarcoma, surgery alone is often curative, and a recurrent tumor occurs in approximately $25 \%$ of the cases. In contrast, an extrauterine Müllerian adenosarcoma recurs in over $50 \%$ of the patients and is associated with a mortality rate of approximately $35 \%$ [6].

The pathogenesis of the extrauterine Müllerian adenosarcoma is unclear. However, the association between exogenous hormonal therapy and the development of malignancy in endometriosis is well known, and the possibility of a neoplastic transformation of endometriotic foci has been suggested by several studies [7, 8]. A large review by Stern of endometriosis-associated neoplasms re-
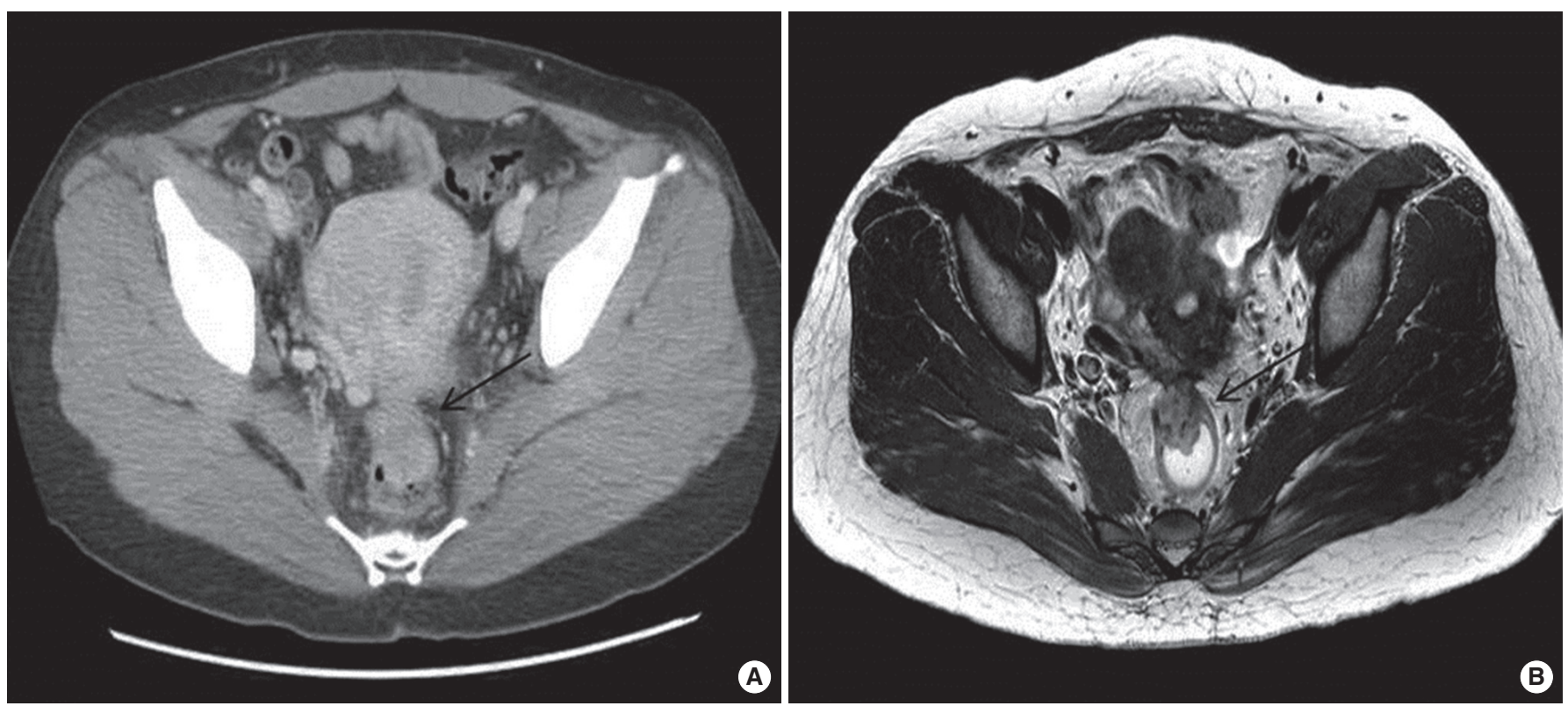

Fig. 2. (A) Abdominal computed tomographic scan showing a 2.7-cm-sized mass (arrow) in the anterior wall of the rectum. (B) Magnetic resonance imaging scan showing a $2.7-\mathrm{cm}$-sized mass (arrow) in the anterior wall of the rectum and intermediate high signal intensity on the T2weighted image. 

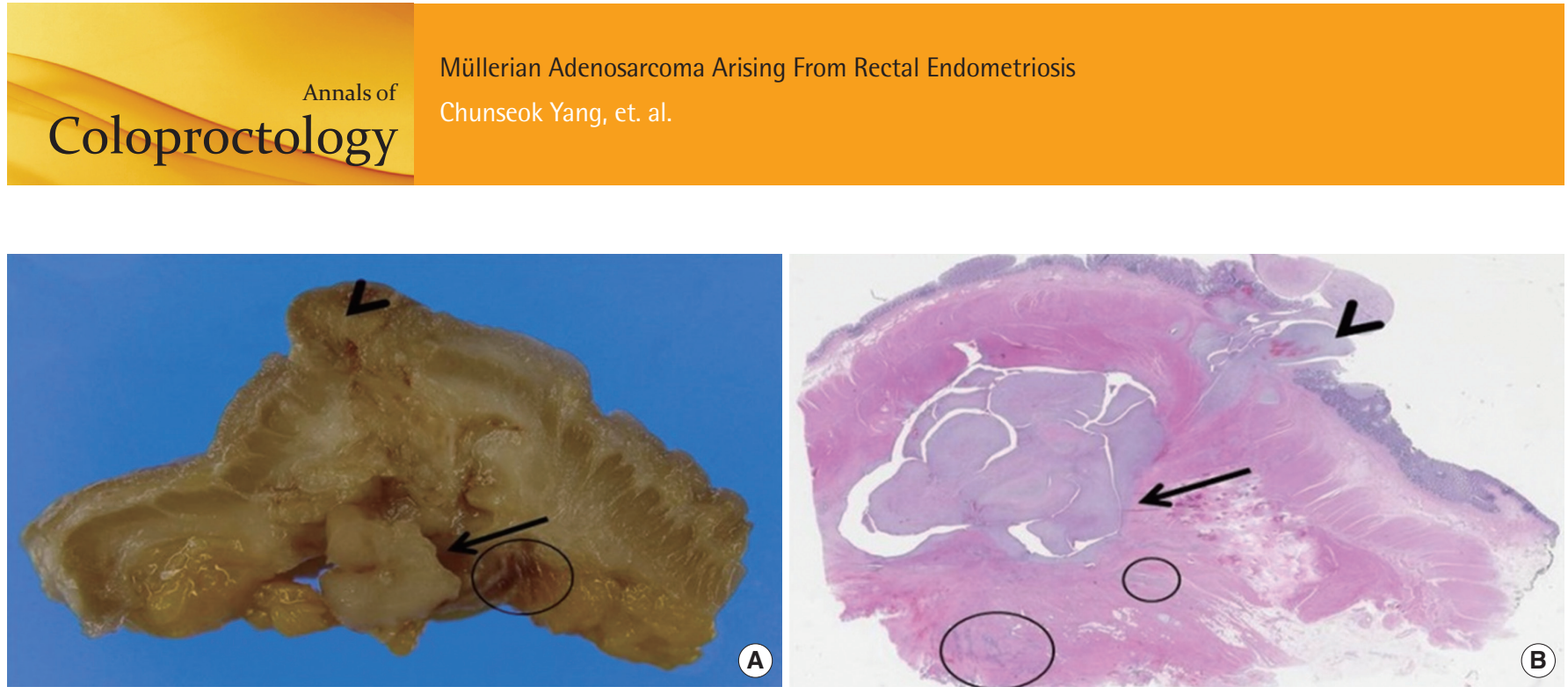

Fig. 3. The excised colon (A) and 1:1 mounted H\&E-stained section (B) show an intramural nodular mass (arrow) with a polypoid mucosal protrusion (arrowhead). Multiple intramural and serosal endometriotic foci (circles) are also present.
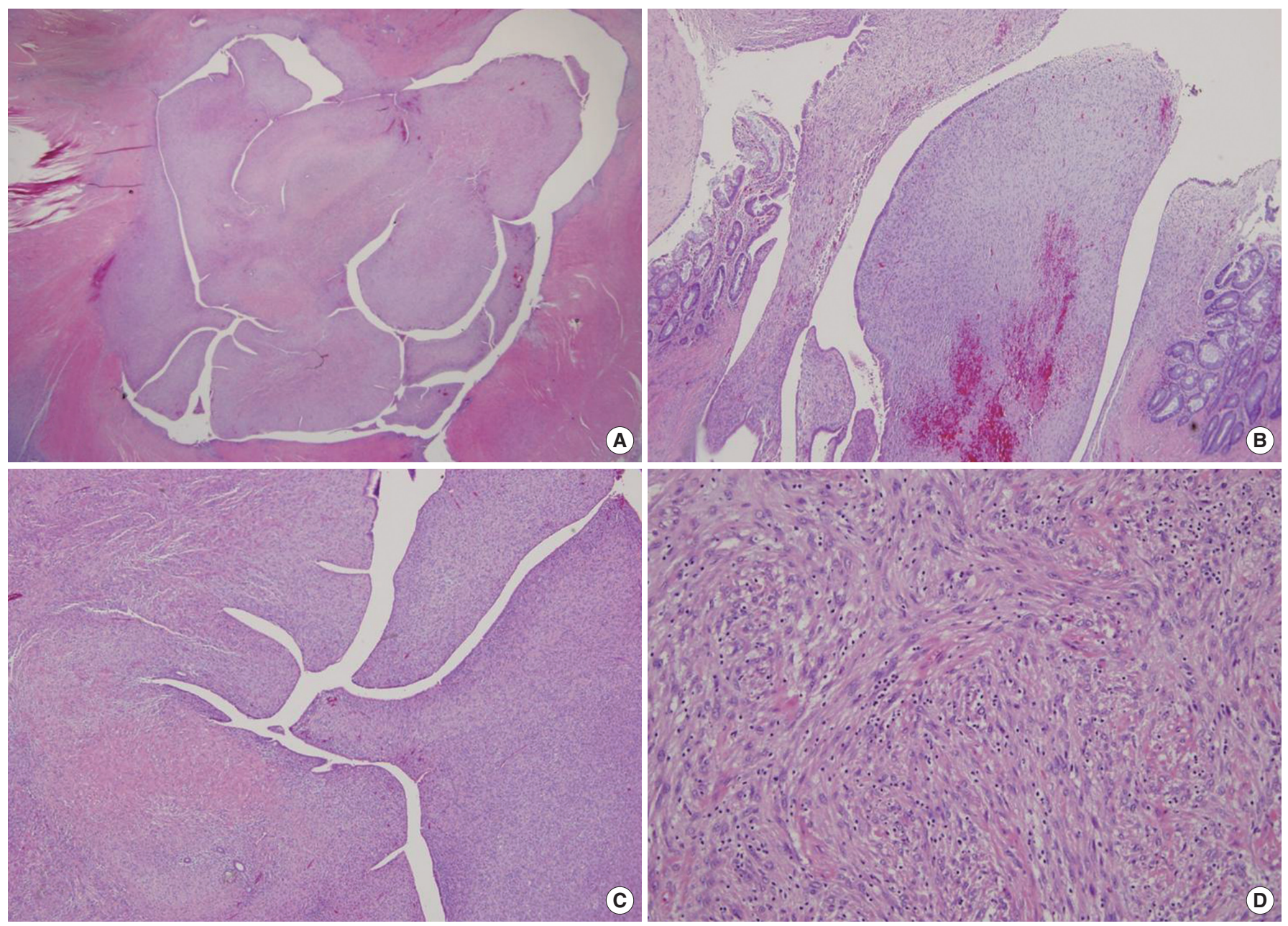

Fig. 4. (A) The intramural nodular mass shows a characteristic biphasic appearance of this neoplasm with a polypoid projection into the glandular space, a typical low power appearance of a phyllodes tumor of the breast (H\&E, $\times 40)$. (B) The mucosal polypoid protrusion of the tumor also show biphasic components $(\mathrm{H} \& \mathrm{E}, \times 100)$. (C) The high-power view of the intramural mass shows a single layer, bland-looking epithelial lining and cellular stroma $(H \& E, \times 100)$. (D) The stromal cells are spindle-like and plump in shape with a fascicular growth pattern and have enlarged nuclei with mild cytologic atypia. A few abnormal mitotic figures are noted. Many inflammatory cells, including lymphocytes, plasma cells and eosinophils, and infiltration are frequently noted throughout the tumor $(\mathrm{H} \& \mathrm{E}, \times 400)$. 

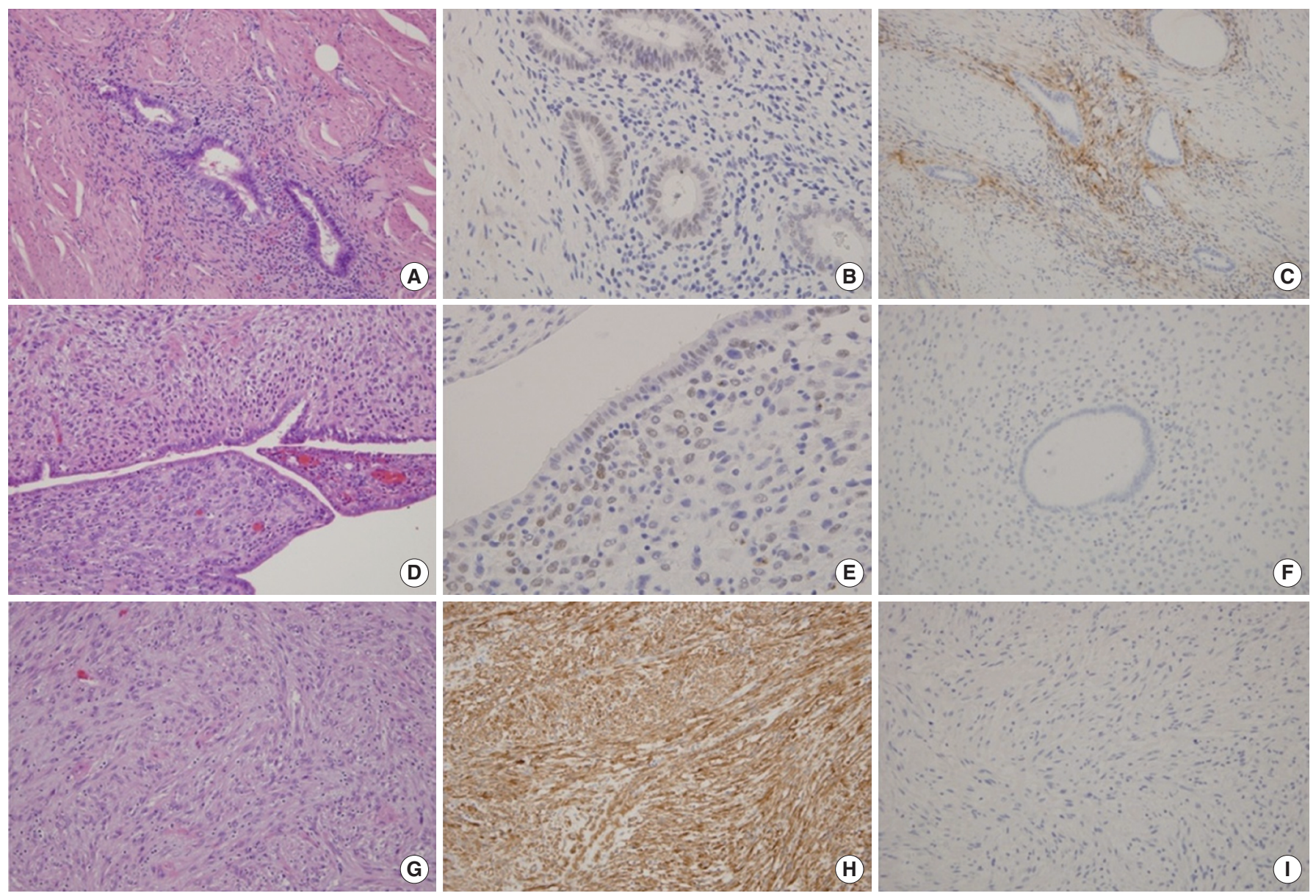

Fig. 5. (A) The endometriotic foci of the wall show tubular endometrial glands with cuffs of endometrial stromal cells (H\&E, $\times 100)$. (B) The endometrial glandular epithelial cells and stromal cells show positive immunoreaction to estrogen receptor (ER) $(\times 200)$. (C) The periglandular endometrial stromal cells show CD10 immunoreactivity $(\times 100)$. (D) The epithelial cell and stromal cells of the intramural mass $(\mathrm{H} \& \mathrm{E}, \times 100)$ shows (E) ER immunoreactivity $(\times 200)$, but not $(\mathrm{F})$ CD10 $(\times 100)$. (G) The tumor stromal cells (H\&E, $\times 200)$ have strong reactions to $(\mathrm{H})$ smooth-muscle actin $(\times 200)$, but not to $(\mathrm{I})$ desmin $(\times 100)$.

vealed that an adenosarcoma was the second most common malignancy after a clear cell carcinoma to arise in the setting of extraovarian endometriosis [7]. Although most tumors associated with endometriosis are of epithelial origin, rare cases of stromal neoplasms, including an adenosarcoma, have been reported and represent $20.8 \%$ of the cases arising in endometriosis from extragonadal sites [9]. This case meets Sampson's criteria for malignant transformation of endometriosis, which include the following: the foci of endometriosis were found in close proximity to the malignancy, the histological appearance was compatible with an endometrial origin, and no other possible primary tumor was seen [10].

First, confirming a histological diagnosis of an adenosarcoma is important. A Müllerian adenosarcoma arising from endometriosis should be distinguished from uncommon benign lesions that are characterized by benign glands, like polypoid endometriosis, adenofibroma, and low-grade endometrial stromal sarcoma with glandular differentiation. The diagnosis could be determined us- ing the criteria recommended by Clement and Scully [1]. They proposed that the diagnosis of an adenosarcoma be made when one or more of the following criteria are present: (1) a stromal mitotic count of two or more mitotic figures per 10 high power field; (2) marked stromal cellular-like periglandular cuffs and intraglandular protrusion of cellular stroma; and (3) more than a mild degree of nuclear atypia of the stromal cells.

Extrauterine tumors are distinctively less common and are primarily located in the pelvic peritoneum, retroperitoneum, broad and round ligaments, vesicouterine pouch, and rectouterine pouch. Until today, only a few cases of an extrauterine Müllerian adenosarcoma arising from endometriosis of the intestine, especially the rectum, have been reported [4]. To our knowledge, this is an extremely rare case of an adenosarcoma arising from endometriosis of the rectum except that a concomitant rectal adenoma and adenosarcoma was reported [11].

The infrequency of a Müllerian adenosarcoma makes establish- 
ing well-defined therapeutic protocols difficult. However, radical surgery remains a therapeutic mainstay. For the treatment of a uterine Müllerian adenosarcoma, a surgical approach similar to that used for the corresponding disease stages of an endometrial carcinoma is recommended, and a total abdominal hysterectomy with a bilateral adnexectomy appears adequate for stage I patients. The decision on giving postoperative chemotherapy may be based on the extent of muscular invasion and sarcomatous growth. Adjuvant chemotherapy is also considered for treating an extrauterine Müllerian adenosarcoma, although its role is still debatable. Although previous reports are inadequate to confirm first-line chemotherapeutic regimens, a few case reports suggest that patients should be treated with liposomal doxorubicin $[6,12]$. The regimen of this recommendation is well tolerated and is effective based on excellent response, minimal toxicity, and convenient dosing schedule in recurrences and adjuvant therapy for extrauterine forms. As the risk of developing recurrent disease was high, a cautionary radiation therapy was given to prevent the probability of a local recurrence. Neoadjuvant or adjuvant pelvic irradiation with chemotherapy was studied in advanced stages, and acceptable outcomes have been reported [13]. In fact, this therapeutic approach was chosen in consideration of the aggressive behavior of the tumor.

The most important negative prognostic factors are lesion location, the degree of myometrial invasion, and sarcomatous overgrowth. In a uterine Müllerian adenosarcoma, the degree of myometrial invasion, sarcomatous overgrowth, and the recurrence rate are strongly correlated to the differentiation grade and the mitotic index of the sarcomatous stromal component [14]. By contrast, a favorable prognostic factor in adenosarcoma patients is the presence of endometriosis [7].

The case we present provides an association with endometriosis, supporting the hypothesis that an extrauterine Müllerian adenosarcoma is transformed from endometriosis. Exogenous or endogenous estrogen may have some role in the transformation process whereas the effectiveness of combined estrogen-progesterone hormone-replacement therapy is still to be determined. Further, the titers of the tumor marker CA 125 were well correlated with the clinical course of endometriosis and might be a predictor for the sarcomatous overgrowth [15].

In conclusion, an extrauterine Müllerian adenosarcoma is rare, but it should be considered in a patient with atypical clinical features or preoperative pathology, especially in a patient with a history of endometriosis. Further research is needed to establish the pathogenesis of an extrauterine adenosarcoma and the therapeutic guidelines.

\section{CONFLICT OF INTEREST}

No potential conflict of interest relevant to this article was reported.

\section{REFERENCES}

1. Clement PB, Scully RE. Müllerian adenosarcoma of the uterus. A clinicopathologic analysis of ten cases of a distinctive type of müllerian mixed tumor. Cancer 1974;34:1138-49.

2. Clement PB, Scully RE. Mullerian adenosarcoma of the uterus: a clinicopathologic analysis of 100 cases with a review of the literature. Hum Pathol 1990;21:363-81.

3. Eichhorn JH, Young RH, Clement PB, Scully RE. Mesodermal (müllerian) adenosarcoma of the ovary: a clinicopathologic analysis of 40 cases and a review of the literature. Am J Surg Pathol 2002; 26:1243-58.

4. Yantiss RK, Clement PB, Young RH. Neoplastic and pre-neoplastic changes in gastrointestinal endometriosis: a study of 17 cases. Am J Surg Pathol 2000;24:513-24.

5. Xie YP, Yao HX, Shen YM. Müllerian adenosarcoma of the uterus with heterologous elements: two case reports and literature review. Arch Gynecol Obstet 2012;286:537-40.

6. Huang GS, Arend RC, Sakaris A, Hebert TM, Goldberg GL. Extragenital adenosarcoma: a case report, review of the literature, and management discussion. Gynecol Oncol 2009;115:472-5.

7. Stern RC, Dash R, Bentley RC, Snyder MJ, Haney AF, Robboy SJ. Malignancy in endometriosis: frequency and comparison of ovarian and extraovarian types. Int J Gynecol Pathol 2001;20:133-9.

8. Higashiura Y, Kajihara H, Shigetomi H, Kobayashi H. Identification of multiple pathways involved in the malignant transformation of endometriosis (Review). Oncol Lett 2012;4:3-9.

9. Irvin W, Pelkey T, Rice L, Andersen W. Endometrial stromal sarcoma of the vulva arising in extraovarian endometriosis: a case report and literature review. Gynecol Oncol 1998;71:313-6.

10. Sampson JA. Endometrial carcinoma of the ovary arising in endometrial tissue in that organ. Arch Surg 1925;10:1-72.

11. Chang HY, Changchien CC, Chen HH, Lin H, Huang CC. Extrauterine müllerian adenosarcoma associated with endometriosis and rectal villotubular adenoma: report of a case and review of the literature. Int J Gynecol Cancer 2005;15:361-5.

12. del Carmen MG, Lovett D, Goodman A. A case of Müllerian adenosarcoma of the uterus treated with liposomal doxorubicin. Gynecol Oncol 2003;88:456-8.

13. Carvalho FM, Carvalho JP, Motta EV, Souen J. Müllerian adenosarcoma of the uterus with sarcomatous overgrowth following tamoxifen treatment for breast cancer. Rev Hosp Clin Fac Med Sao Paulo 2000;55:17-20.

14. Verschraegen CF, Vasuratna A, Edwards C, Freedman R, Kudelka AP, Tornos $\mathrm{C}$, et al. Clinicopathologic analysis of mullerian adenosarcoma: the M.D. Anderson Cancer Center experience. Oncol Rep 1998;5:939-44.

15. Inoue M, Fukuda $\mathrm{H}$, Tanizawa $\mathrm{O}$. Adenosarcomas originating from sites other than uterine endometrium. Int J Gynaecol Obstet 1995;48:299-306. 